\title{
Pediatric silent corticotroph pituitary adenoma and role for proton therapy: case report
}

\author{
Nicole Prendergast, BS, ${ }^{1}$ Philipp R. Aldana, MD, ${ }^{2}$ Ronny L. Rotondo, MD, CM, ${ }^{3}$ \\ Lournaris Torres-Santiago, MD, ${ }^{4}$ and Alexandra D. Beier, DO ${ }^{2}$
}

\begin{abstract}
${ }^{1}$ Frank H. Netter MD School of Medicine, Quinnipiac University, North Haven, Connecticut; '2Division of Pediatric Neurosurgery, UF Health, Jacksonville; ${ }^{3}$ Department of Radiation Oncology, University of Florida, Gainesville; and ${ }^{4}$ Division of Pediatric Endocrinology, Diabetes and Metabolism, Nemours Children's Health System, Jacksonville, Florida
\end{abstract}

\begin{abstract}
Tumors involving the sella are commonly craniopharyngiomas, optic pathway gliomas, or pituitary adenomas. Functioning adenomas are expected, with prolactinomas topping the differential. The authors present the case of a silent corticotroph adenoma, which has not been described in the pediatric population, and they detail the use of proton therapy, which is also novel.
\end{abstract}

https://thejns.org/doi/abs/10.3171/2018.9.PEDS18107

KEYWORDS pediatric; brain tumor; pituitary adenoma; proton therapy; pituitary surgery

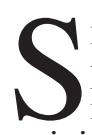
ILENT corticotroph adenomas (SCAs) are a distinct pathological type of nonfunctioning pituitary adenoma defined by their positive immunohistochemical staining for adrenocorticotrophic hormone (ACTH) and normal cortisol levels in the absence of Cushing disease. These adenomas typically occur in the adult population and constitute approximately $5 \%-6 \%$ of nonfunctional pituitary adenomas. Although the pathophysiology of SCA is poorly understood, these tumors have been characterized by more aggressive behavior and higher recurrence rates. ${ }^{1,2}$

Pituitary tumors are rare in the pediatric population, with the majority of tumors being functional adenomas., ${ }^{9,11}$ Here, we describe a child with SCA. To our knowledge, SCA in the pediatric population has not been described in the literature, nor has the use of proton therapy for SCA been described.

\section{Case Report}

History and Examination

An otherwise healthy and developmentally normal 13-year-old boy with a 1-year history of headaches pre- sented to his primary care physician with severe headache, nausea, and vomiting. Persistence of this headache prompted a CT scan, which revealed a large suprasellar lesion with resultant hydrocephalus. Upon retrospective review of his growth charts, it was found that he had a gradual growth deceleration, starting at the age of 12 years, from the 25th to the 3rd percentile. Otherwise, he was neurologically intact. Subsequent MRI revealed a 2.9 $\times 2.8 \times 5.5-\mathrm{cm}$ lobulated, enhancing sellar and suprasellar mass extending superiorly and obstructing the bilateral foramina of Monro, leading to mild to moderate enlargement of the lateral ventricles and transependymal edema (Fig. 1). Differential diagnosis included craniopharyngioma, pituitary adenoma, and hypothalamic-chiasmatic astrocytoma. Laboratory tests of pituitary function were within normal limits with regard to thyroid function tests (TFTs), ACTH, cortisol, follicle-stimulating hormone (FSH), and luteinizing hormone (LH) but revealed low levels of insulin-like growth factor-1 (IGF-1; $149 \mathrm{ng} / \mathrm{ml}$, $-2.1 \mathrm{SD})$ and testosterone $(28 \mathrm{ng} / \mathrm{dl})$. The presence of mildly elevated prolactin levels $(45.3 \mathrm{ng} / \mathrm{ml})$, rather than the higher levels exceeding $200 \mathrm{ng} / \mathrm{ml}$ that is characteristic of prolactinomas, was attributed to the stalk effect.

ABBREVIATIONS $\mathrm{ACTH}=$ adrenocorticotrophic hormone; $\mathrm{CTV}=$ clinical target volume; $\mathrm{EVD}=$ external ventricular drain; $\mathrm{FSH}=$ follicle-stimulating hormone; $\mathrm{GH}=$ growth hormone; IGF-1 = insulin-like growth factor-1; NFA = nonfunctioning adenoma; RT = radiation therapy; SCA = silent corticotroph adenoma.

SUBMITTED April 17, 2018. ACCEPTED September 12, 2018.

INCLUDE WHEN CITING Published online November 16, 2018; DOI: 10.3171/2018.9.PEDS18107. 

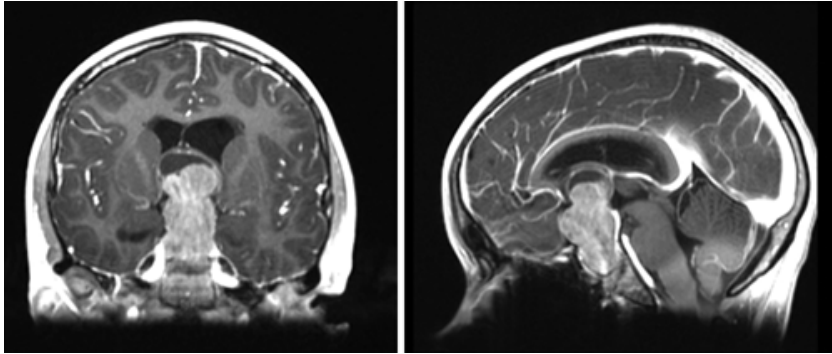

FIG. 1. Coronal (left) and sagittal (right) MR images with contrast revealing an enhancing sellar and suprasellar mass.

In-hospital preoperative ophthalmology testing revealed hereditary color vision deficiency but no visual field deficit, no papilledema, full extraocular muscle movement, and $20 / 20$ vision.

\section{Operative Course}

To help manage the hydrocephalus and determine the definitive management of the tumor, an endoscopic biopsy, a septostomy, and external ventricular drain (EVD) placement were performed via a right burr hole craniectomy. Pathological results revealed a pituitary adenoma, and the patient underwent a staged subtotal endoscopic endonasal resection of the pituitary adenoma (Fig. 2). Resection was limited because of the patient's immature sinus anatomy and the firmness of the tumor. The patient tolerated the EVD weaning, and the drain was removed 6 days following the endonasal resection. Cerebrospinal fluid leakage along with intracranial hypertension symptoms required replacement of the EVD; however, this occurred together with the development of hyponatremia from cerebral salt wasting. The hyponatremia was treated with hypertonic saline and sodium supplements, and his sodium normalized. Imaging revealed stable ventricles, and the EVD could be removed without complications.

Microscopic examination of the sellar and suprasellar biopsy specimens showed a monomorphic population of pituitary cells with a fragmented and decreased reticulin network. Immunohistochemical stains revealed strong ACTH expression, whereas prolactin, FSH, and thyroidstimulating hormone (TSH) were negative. The proliferative index was low. Keratin and epithelial membrane antigen within the tumor cells were positive and negative,
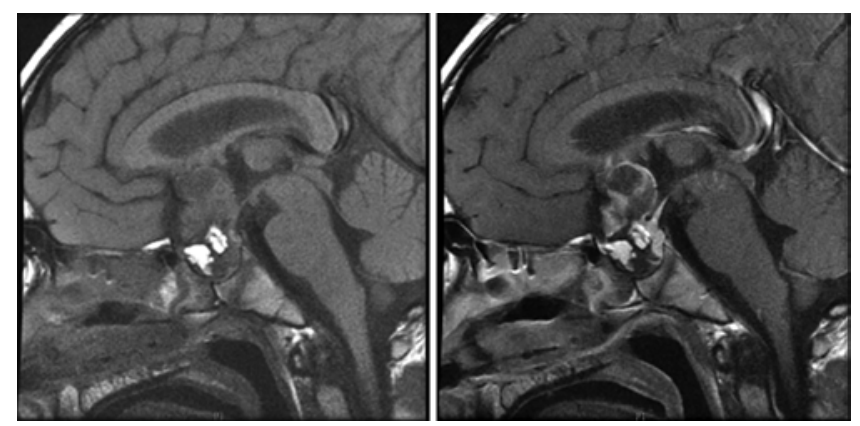

FIG. 2. Precontrast (left) and postcontrast (right) sagittal MR images demonstrating subtotal resection of a pituitary adenoma.

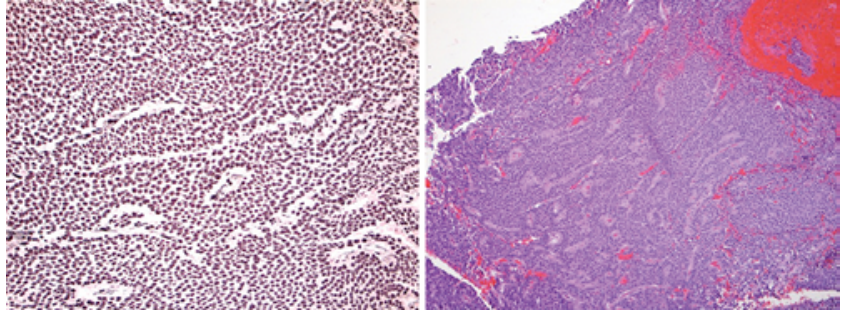

FIG. 3. Left: Reticulin staining revealed a decreased reticulin network. Original magnification $\times 20$. Right: Silent corticotroph adenoma. H \& E staining revealed a monomorphic population of pituitary cells. Original magnification $\times 10$. Figure is available in color online only.

respectively. Strong ACTH expression in the absence of clinical or biochemical evidence of Cushing disease suggested an SCA (Fig. 3). Therefore, given the aggressive nature of SCA, we determined that further resection of the residual tumor should be performed. The patient underwent a subfrontal craniotomy for resection of the residual sellar and suprasellar components of the tumor. Intraoperative MRI did not conclusively demonstrate residual tumor. The patient was neurologically intact when he was discharged on postoperative day 5 .

Surveillance MRI performed 4 months later demonstrated a tumor measuring $9 \times 5 \mathrm{~mm}$ in the right cavernous sinus inferomedial to the right internal carotid artery (Fig. 4). Thus, he was referred for adjuvant radiation therapy (RT) and ultimately completed a course of postoperative passively scattered 3D conformal proton therapy to a dose of 45 cobalt gray equivalent (CGE) at $1.8 \mathrm{CGE}$ per daily fraction for a total of 25 fractions delivered over 5 weeks, using daily in-room $\mathrm{kV}$ x-ray image guidance (Fig. 5). Proton therapy was initiated approximately 5.5 months following the last surgery. Pre- and postoperative

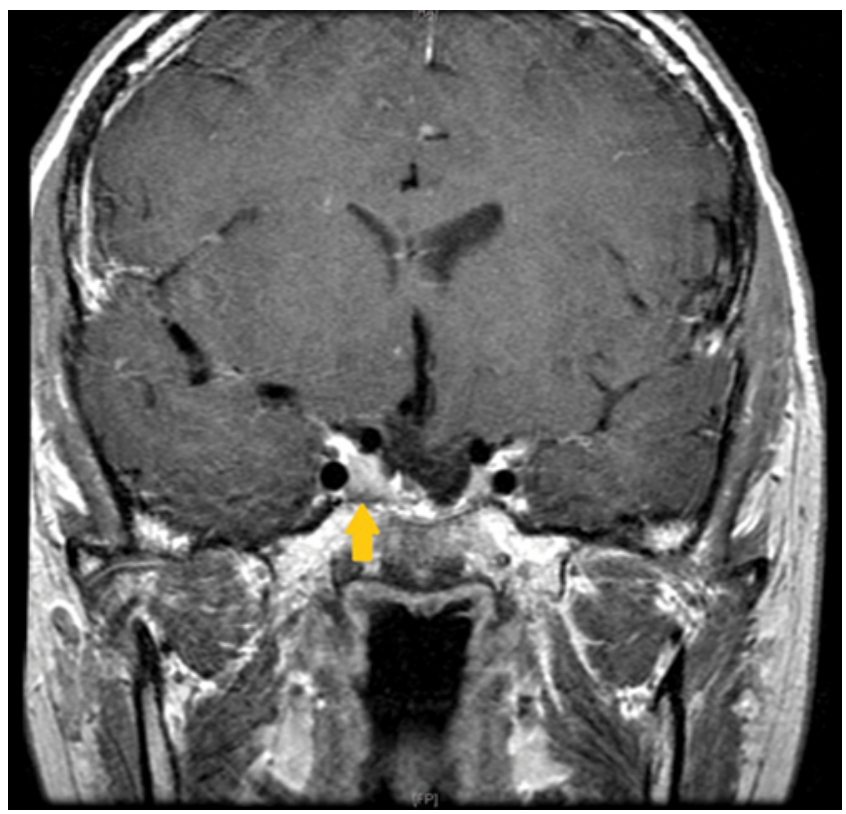

FIG. 4. Postoperative coronal MR image depicting residual disease (arrow) in the right cavernous sinus. Figure is available in color online only. 


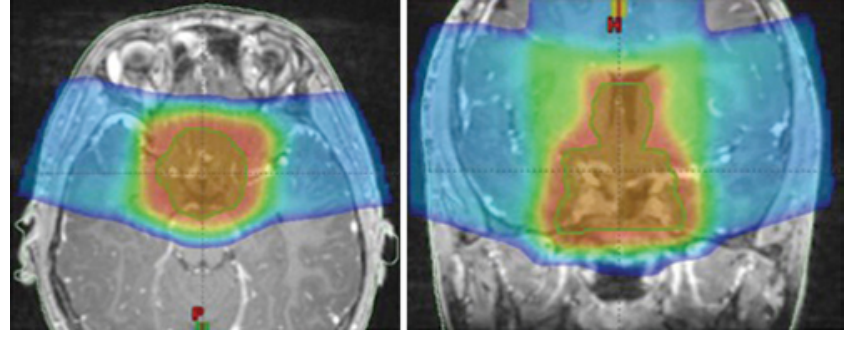

FIG. 5. Axial (left) and coronal (right) representative dose distributions for a passively scattered 3D conformal proton therapy plan. Figure is available in color online only.

MRI studies were used for target definition. Gross tumor volume (GTV) was defined as the gross residual disease in the cavernous sinus. The clinical target volume (CTV) was defined as the GTV plus a small, customized margin $(5 \mathrm{~mm})$ plus the resection bed. The planning target volume (PTV) consisted of a 3-mm geometrical expansion of the CTV. The PTV was treated using a three-field arrangement, and the plan was normalized so that $99 \%$ of the CTV received $100 \%$ of the dose. The patient tolerated the proton therapy well without unexpected acute adverse effects. Subsequent MRI at 6 months postoperatively/1 month post-RT, 9 months postoperatively $/ 3$ months post$\mathrm{RT}$, and 12 months postoperatively/6 months post-RT revealed stable cavernous sinus disease. At the 18-month postoperative/12-month post-RT MRI evaluation, the cavernous sinus disease had decreased with minimal residual disease. This has been stable for 30 months postoperatively/24 months post-RT (Fig. 6).

From an endocrinological standpoint, postoperatively the patient showed improvement in IGF-1 (190 ng/ml, $-1.5 \mathrm{SD})$ and testosterone levels (178 $\mathrm{ng} / \mathrm{dl})$. Monitoring of pituitary functions was done every 3 months. Despite normal pubertal progression (peak testosterone $725 \mathrm{ng} /$ dl), IGF-1 levels did not increase and growth velocity remained below $5 \mathrm{~cm} / \mathrm{yr}$, translating into a diagnosis of partial growth hormone (GH) deficiency. The patient's bone age was 14 years 6 months for a chronological age of 13 years 6 months. Predicted adult height was approximately 61 inches compared to a midparental height at the 10th percentile. He was briefly treated with GH (IGF-1 $426 \mathrm{ng}$ / $\mathrm{ml}$ ) and anastrozole starting 12 months after craniotomy. He had an excellent initial response, but adherence to treatment became suboptimal and the patient discontinued treatment. He reached a final adult height of 61.5 inches. At the age of 16 years, his IGF-1 level was $81 \mathrm{ng} / \mathrm{ml}$, and $\mathrm{GH}$ was restarted using adult transition dosing. Prolactin levels have remained slightly elevated $(17.7-24.5 \mathrm{ng} / \mathrm{ml})$ but stable. He developed partial central hypothyroidism 5 months after craniotomy, which was successfully treated with thyroid hormone replacement. Morning cortisol levels were normal until 10 months postoperatively when he had a low morning cortisol $(7.7 \mu \mathrm{g} / \mathrm{dl})$ as compared to previous levels (13.6-20.3 $\mu \mathrm{g} / \mathrm{dl})$. Low-dose ACTH stimulation testing was consistent with partial adrenal insufficiency (peak level $14.9 \mu \mathrm{g} / \mathrm{dl}$ at 45 minutes). He has not developed diabetes insipidus.

On neuroophthalmological exams at 6 months post-

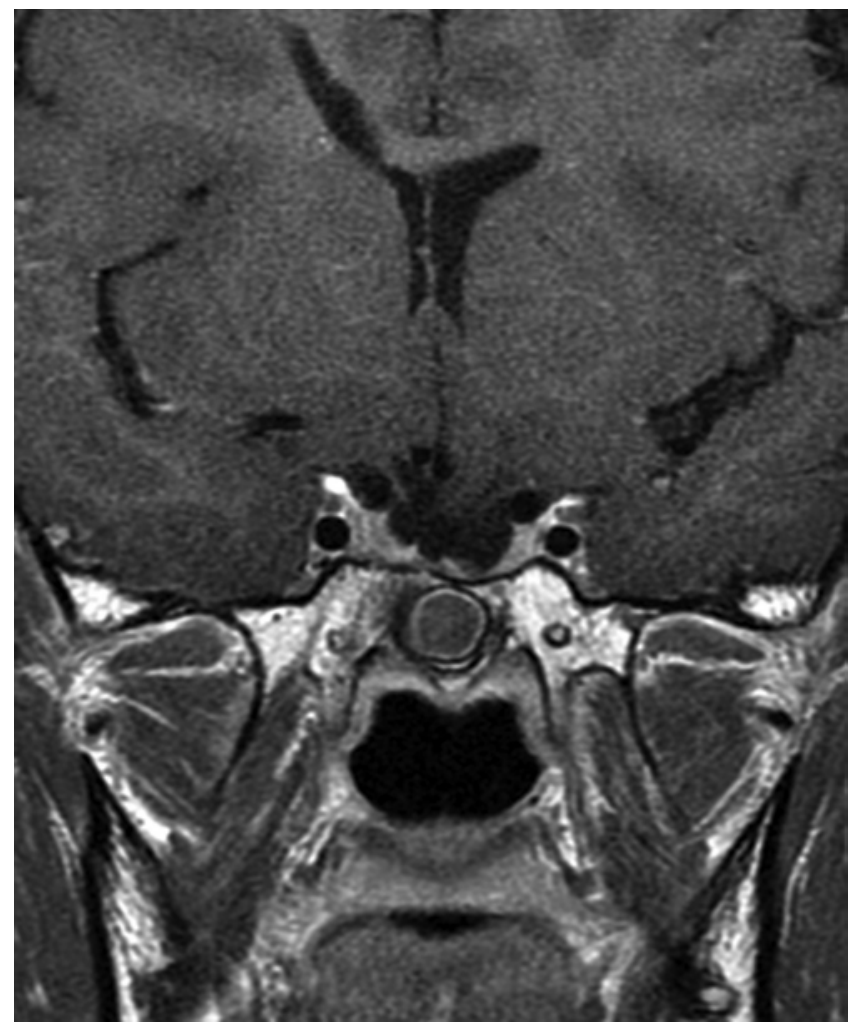

FIG. 6. Post-proton therapy coronal MR image revealing stable minimal residual right cavernous sinus disease.

operatively, he was noted to have mild to moderate optic nerve atrophy and temporal pallor on the left. In addition, on Goldmann visual field testing, he had temporal field loss on the right and superior temporal quadrantanopia with an enlarged blind spot on the left. These findings were thought to be attributable to the tumor size itself and have remained stable on serial examinations.

\section{Discussion}

Pituitary tumors are rare in the pediatric population with a prevalence of up to 1 case per 1 million children. ${ }^{11}$ Craniopharyngiomas and pituitary adenomas are the two main types of tumors located in the sellar region in pediatric patients, with craniopharyngiomas accounting for $80 \%-90 \%$ of tumors in the pituitary fossa. Pituitary adenomas are less frequent and are generally functional adenomas with prolactinomas being the most common. ${ }^{9,11}$ ACTH-secreting tumors in the pediatric population are more rare; when they present, however, they are most common in early childhood, whereas prolactinomas are more prevalent in older children and adolescents. ${ }^{9}$ Other tumors in the vicinity that can be present similarly and should be included in the differential diagnosis are optic pathway and hypothalamic chiasmatic gliomas. ${ }^{6}, 15$ Silent corticotroph adenomas are extremely rare and have not been described in the pediatric literature. To reach the diagnosis of SCA, there must be strong immunohistochemical positivity for ACTH but a lack of biochemical and clinical evidence of hypercortisolism. ${ }^{13,16}$ Furthermore, the biopsy must reveal 
ACTH to be the dominant hormone expressed rather than prolactin, gonadotroph, thyrotroph, or growth hormones. ${ }^{2}$ A pituitary database searched for the period from 1990 to 2000 included only 15 patients with SCAs based on biochemical and clinical evidence, which included visual disturbances and headaches, with visual disturbances being the most common. ${ }^{2,3}$ Of these 15 patients, 10 were male and 5 were female, with a mean age of 50.8 years (range 35-65 years). Treatment for recurrence was required in 5 cases, with 2 patients succumbing to their disease. ${ }^{2}$

Because of their higher propensity for recurrence, SCAs have been cited for their aggressive and transforming behavior requiring greater attention to follow-up and surgical treatment. Investigation of the markers related to cell proliferation, invasiveness, motility, and migration, notably osteopontin, matrix metalloproteinases (MMPs), and fibroblast growth factor receptors (FGFRs), could explain aggressiveness in SCA cases. ${ }^{14}$

SCAs have also shown transformation of secretion patterns and unpredictable postoperative behavior. Vaughan et al. and Cooper et al. were the first to report the development of Cushing disease from previously silent adenomas, that is, SCAs. ${ }^{7,16}$ A 2015 review of patients with pituitary adenomas, 44 SCAs and 132 cases of Cushing disease, revealed 5 cases with clinical and biochemical transformations of the corticotrophic tumors. ${ }^{17}$ Three tumor transformations from SCA to Cushing disease were seen, and another tumor converted from Cushing disease to SCA. The fifth case involved a transition to Cushing disease, like the first 3 cases, followed by another transition back to SCA. Two of these 5 patients underwent radical resections, and all but one case involved multiple surgeries. The final result was a shift from ACTH-silent to functioning adenomas in $4(9 \%)$ of the 44 SCA cases and reversion to a silent corticotroph adenoma in $2(1.5 \%)$ of the 132 Cushing disease cases. Theories for the cause of clinically silent ACTH secretion in cases of SCA include decreased biological activity of the ACTH secreted by the tumor or modification of the ACTH's molecular structure by the tumor. ${ }^{2}$ In cases in which initially identified ACTH produced by SCA has become active and indicative of Cushing disease, it has been hypothesized that previously inactive ACTH may have become active or the release mechanism is more effective following the initial diagnosis. ${ }^{2}$ Not only have transitions to Cushing disease been seen in cases of SCA, but Brown et al. have also described a case of carcinoma development from SCA. ${ }^{4}$

In comparing SCAs to nonfunctioning adenomas (NFAs), Cooper et al. showed that SCAs had a 5-year recurrence rate of $63 \%$ compared to $38 \%$ for NFAs. ${ }^{8,14}$ A more recent retrospective analysis on 33 SCA cases showed a higher cavernous sinus invasion rate $(45.5 \%$ vs $30.2 \%$, respectively) and greater residual tumor progression $(24.2 \%$ vs $11.1 \%$, respectively) upon reevaluation, compared to ACTH-negative tumors. ${ }^{10}$

Contrary to these results, Bradley et al. found a similar recurrence rate in cases of SCA and NFA. There was only a $32 \%$ recurrence in those with ACTH-positive tumors, compared to a $33 \%$ recurrence rate in the non-ACTH group. ${ }^{3}$ Similarly, of 28 SCAs and 134 cases of nonfunctioning pituitary adenomas without $\mathrm{ACTH}$ reactivity, Cho et al. showed recurrence rates of $25 \%$ and $26.9 \%$, respectively. ${ }^{5}$ Despite the absence of significant differences in recurrence rates between the SCA and NFA groups, in those cases that did recur, both Bradley et al. and Cho et al. saw more SCA cases with multiple recurrences and in less time than with the NFAs. ${ }^{3,5}$ Furthermore, SCAs were found to be more aggressive than the NFA counterparts, exhibiting multiple episodes of regrowth in some patients. ${ }^{3}$ Unfortunately, the mechanism for this behavior was not examined further in either study.

Upon a review of the literature, we found that the type and degree of resection do not appear to be indicators of regrowth., $3,5,14,16$ The primary adjuvant treatment option for SCA is radiotherapy, including fractionated (30-50 Gy) and stereotactic radiosurgery. Nonendocrine late effects of radiotherapy include a risk of second tumors within the radiation field, visual impairment, and neurocognitive deficits. More recent advances in radiation techniques and modalities, including proton therapy, provide the promise of reduced late effects with high rates of local control. Kennedy et al. recently reported on the early results of proton therapy for pituitary adenomas at the University of Florida Health Proton Therapy Institute. ${ }^{12}$ Their retrospective study included 17 patients treated with passively scattered proton therapy, and the primary reason for referral was cavernous sinus invasion (15 patients). With a median follow-up of 3.9 years, local control, progressionfree survival, and cause-specific survival at 3 years were all $100 \%$. Compared to intensity-modulated radiotherapy (IMRT), proton therapy significantly reduced the mean dose to the brain, both temporal lobes, and hippocampi. This lower dose to healthy brain may reduce the neurocognitive impact of RT, and the lower integral dose may also reduce the risk of secondary tumors.

Given the aggressive growth pattern and risk of neurological morbidity, especially vision impairment, with tumor progression, all relevant parties elected to treat this patient with adjuvant proton RT in order to maximize the chance of long-term local control while trying to minimize the risk of the long-term late effects of treatment. This is the first pediatric as well as the first adult case in which proton therapy has been utilized for adjuvant treatment for SCA. This patient will continue to be monitored, as longer-term follow-up is warranted regarding his endocrinological and tumor status.

\section{Conclusions}

We present the first report of a pediatric patient with SCA, as well as the first SCA treated with proton therapy. The described aggressive nature, possible increased propensity for recurrence, greater tendency for invasion, transforming nature of adult SCA, as well as the lack of conclusive studies, indicate the need for close biochemical, clinical, and neuroradiological follow-up. The longterm outcome of proton therapy for SCA is unknown; however, the benefits of reducing nonendocrine side effects are paramount in the pediatric population.

\section{Acknowledgments}

We appreciate the assistance of Dr. Bradley Cheek, Southeast- 
ern Pathology Associates, in the evaluation of this patient's pathological slides. We also thank Kelsey Hayward for her assistance in manuscript preparation.

\section{References}

1. Alahmadi H, Lee D, Wilson JR, Hayhurst C, Mete O, Gentili F, et al: Clinical features of silent corticotroph adenomas. Acta Neurochir (Wien) 154:1493-1498, 2012

2. Baldeweg SE, Pollock JR, Powell M, Ahlquist J: A spectrum of behaviour in silent corticotroph pituitary adenomas. Br J Neurosurg 19:38-42, 2005

3. Bradley KJ, Wass JAH, Turner HE: Non-functioning pituitary adenomas with positive immunoreactivity for ACTH behave more aggressively than ACTH immunonegative tumours but do not recur more frequently. Clin Endocrinol (Oxf) 58:59-64, 2003

4. Brown RL, Wollman R, Weiss RE: Transformation of a pituitary macroadenoma into to a corticotropin-secreting carcinoma over 16 years. Endocr Pract 13:463-471, 2007

5. Cho HY, Cho SW, Kim SW, Shin CS, Park KS, Kim SY: Silent corticotroph adenomas have unique recurrence characteristics compared with other nonfunctioning pituitary adenomas. Clin Endocrinol (Oxf) 72:648-653, 2010

6. Collins VP, Jones DTW, Giannini C: Pilocytic astrocytoma: pathology, molecular mechanisms and markers. Acta Neuropathol 129:775-788, 2015

7. Cooper ME, Murray RM, Kalnins R, Woodward J, Jerums $\mathrm{G}$ : The development of Cushing's syndrome from a previously silent pituitary tumour. Aust N Z J Med 17:249-251, 1987

8. Cooper O, Ben-Shlomo A, Bonert V, Bannykh S, Mirocha J, Melmed S: Silent corticogonadotroph adenomas: clinical and cellular characteristics and long-term outcomes. Horm Cancer 1:80-92, 2010

9. Guaraldi F, Storr HL, Ghizzoni L, Ghigo E, Savage MO: Paediatric pituitary adenomas: a decade of change. Horm Res Paediatr 81:145-155, 2014

10. Ioachimescu AG, Eiland L, Chhabra VS, Mastrogianakis GM, Schniederjan MJ, Brat D, et al: Silent corticotroph adenomas: Emory University cohort and comparison with ACTH-negative nonfunctioning pituitary adenomas. Neurosurgery 71:296-304, 2012

11. Keil MF, Stratakis CA: Pituitary tumors in childhood: up- date of diagnosis, treatment and molecular genetics. Expert Rev Neurother 8:563-574, 2008

12. Kennedy WR, Dagan R, Rotondo RL, Louis D, Morris CG, Indelicato DJ: Proton therapy for pituitary adenoma. Appl Radiat Oncol 90:299, 2014

13. Mayson SE, Snyder PJ: Silent (clinically nonfunctioning) pituitary adenomas. J Neurooncol 117:429-436, 2014

14. Mete $\mathrm{O}$, Hayhurst $\mathrm{C}$, Alahmadi H, Monsalves E, Gucer H, Gentili F, et al: The role of mediators of cell invasiveness, motility, and migration in the pathogenesis of silent corticotroph adenomas. Endocr Pathol 24:191-198, 2013

15. Patibandla MR, Thotakura AK, Uppin M, Challa S, Addagada GC, Nukavarapu M: Parietal pilomyxoid astrocytoma with recurrence in 10 months: a case report and review of literature. Asian J Neurosurg 11:323, 2016

16. Vaughan NJ, Laroche CM, Goodman I, Davies MJ, Jenkins JS: Pituitary Cushing's disease arising from a previously non-functional corticotrophic chromophobe adenoma. Clin Endocrinol (Oxf) 22:147-153, 1985

17. Zoli M, Faustini-Fustini M, Mazzatenta D, Marucci G, De Carlo E, Bacci A, et al: ACTH adenomas transforming their clinical expression: report of 5 cases. Neurosurg Focus 38(2):E15, 2015

\section{Disclosures}

The authors report no conflict of interest concerning the materials or methods used in this study or the findings specified in this paper.

\section{Author Contributions}

Conception and design: Beier. Acquisition of data: all authors. Analysis and interpretation of data: all authors. Drafting the article: all authors. Critically revising the article: all authors. Reviewed submitted version of manuscript: all authors. Approved the final version of the manuscript on behalf of all authors: Beier. Study supervision: Beier.

\section{Correspondence}

Alexandra D. Beier: UF Health, Jacksonville, FL. alexandra. beier@jax.ufl.edu. 